\title{
Podocyte-specific Nox4 deletion affords renoprotection in a mouse model of diabetic nephropathy
}

\author{
Jay C. Jha ${ }^{1,2}$ • Vicki Thallas-Bonke ${ }^{1}$. Claudine Banal ${ }^{1} \cdot$ Stephen P. Gray $^{1}$. \\ Bryna S. M. Chow ${ }^{1}$ - Georg Ramm ${ }^{3}$ - Susan E. Quaggin ${ }^{4}$ - Mark E. Cooper ${ }^{1,2}$. \\ Harald H. H. W. Schmidt ${ }^{5}$. Karin A. Jandeleit-Dahm ${ }^{1,2}$
}

Received: 6 July 2015 / Accepted: 1 October 2015 /Published online: 28 October 2015

(C) Springer-Verlag Berlin Heidelberg 2015

\begin{abstract}
Aims/hypothesis Changes in podocyte morphology and function are associated with albuminuria and progression of diabetic nephropathy. NADPH oxidase 4 (NOX4) is the main source of reactive oxygen species (ROS) in the kidney and Nox4 is upregulated in podocytes in response to high glucose. We assessed the role of NOX4-derived ROS in podocytes in vivo in a model of diabetic nephropathy using a podocyte-specific NOX4-deficient mouse, with a major focus on the development of albuminuria and ultra-glomerular structural damage.

Methods Streptozotocin-induced diabetes-associated changes in renal structure and function were studied in male floxedNox4 and podocyte-specific, NOX4 knockout
\end{abstract}

Harald H. H. W. Schmidt and Karin A. Jandeleit-Dahm contributed equally as senior authors.

Electronic supplementary material The online version of this article (doi:10.1007/s00125-015-3796-0) contains peer-reviewed but unedited supplementary material, which is available to authorised users.

Karin A. Jandeleit-Dahm

karin.jandeleit-dahm@bakeridi.edu.au

1 Diabetes Complications Division, Baker IDI Heart \& Diabetes Research Institute, PO Box 6492, St Kilda Rd,

Melbourne, VIC 8008, Australia

2 Department of Medicine, Monash University, Melbourne, VIC, Australia

3 Monash Micro-imaging, Monash University, Melbourne, VIC, Australia

4 Robert H. Lurie Medical Research Center, Chicago, IL, USA

5 Department of Pharmacology, Cardiovascular Research Institute Maastricht (CARIM), Faculty of Medicine, Health \& Life Science, Maastricht University, Maastricht, the Netherlands
(podNox4KO) mice. We assessed albuminuria, glomerular extracellular matrix accumulation and glomerulosclerosis, and markers of ROS and inflammation, as well as glomerular basement membrane thickness, effacement of podocytes and expression of the podocyte-specific protein nephrin.

Results Podocyte-specific Nox4 deletion in streptozotocininduced diabetic mice attenuated albuminuria in association with reduced vascular endothelial growth factor (VEGF) expression and prevention of the diabetes-induced reduction in nephrin expression. In addition, podocyte-specific Nox4 deletion reduced glomerular accumulation of collagen IV and fibronectin, glomerulosclerosis and mesangial expansion, as well as glomerular basement membrane thickness. Furthermore, diabetes-induced increases in renal ROS, glomerular monocyte chemoattractant protein-1 (MCP-1) and protein kinase $\mathrm{C}$ alpha (PKC- $\alpha$ ) were attenuated in podocyte-specific NOX4-deficient mice. Conclusions/interpretation Collectively, this study shows the deleterious effect of Nox4 expression in podocytes by promoting podocytopathy in association with albuminuria and extracellular matrix accumulation in experimental diabetes, emphasising the role of NOX4 as a target for new renoprotective agents.

Keywords Albuminuria · Diabetic nephropathy · Glomerular basement membrane $\cdot$ NADPH oxidase $4 \cdot$ Podocyte $\cdot$

Reactive oxygen species

$\begin{array}{ll}\text { Abbreviations } \\ \text { ACR } & \text { Albumin/creatinine ratio } \\ \text { DN } & \text { Diabetic nephropathy } \\ \text { ECM } & \text { Extracellular matrix } \\ \text { GBM } & \text { Glomerular basement membrane } \\ \text { 8-OHdG } & \text { 8-Hydroxy-2'-deoxyguanosine }\end{array}$




$\begin{array}{ll}\text { MCP } & \text { Monocyte chemoattractant protein } \\ \text { NOX } & \text { NADPH oxidase } \\ \text { PKC } & \text { Protein kinase C } \\ \text { ROS } & \text { Reactive oxygen species } \\ \text { VEGF } & \text { Vascular endothelial growth factor }\end{array}$

\section{Introduction}

Diabetic nephropathy (DN) is the leading cause of renal failure in the world, with patients often requiring dialysis or kidney transplantation $[1,2]$. Podocyte damage and loss have been suggested to play pivotal roles in the pathogenesis of DN including albuminuria, which is a key feature of this condition $[3,4]$. Podocytes are glomerular visceral epithelial cells connected by the slit diaphragm, which functions as a sizeselective filtration barrier [5]. A number of studies have identified 'oxidative stress' as a crucial mediator in the development and progression of DN [6-8]. It has been postulated that this enhanced oxidative stress via the pro-oxidant enzyme NADPH oxidase (NOX), particularly NOX4 [7, 9, 10] and more recently NOX5 [11], in the diabetic kidney causes injury to podocytes and is associated with deleterious changes in renal function and structure [4, 12-14].

In a recent study, we found that global genetic deletion or pharmacological inhibition of NOX4 significantly attenuated diabetes-induced increases in albuminuria, glomerulosclerosis and accumulation of extracellular matrix (ECM) proteins via a reduction in reactive oxygen species (ROS) production in an experimental model of DN [9]. It has been suggested that stimuli such as high glucose, TGF- $\beta$ and angiotensin II increase ROS production and lead to injury and apoptosis in podocytes, and are associated with increased excretion of albumin into the urine [4, 15-17]. Another study has reported that systemic administration of a nonspecific NOX inhibitor, apocynin, ameliorated urinary albumin excretion in a diabetic mouse model $[18,19]$. Recently, we found that silencing NOX4 using short hairpin RNA or inhibiting NOX4 using a novel, specific NOX1/NOX4 inhibitor (GKT137831) in human podocytes significantly reduced the high glucoseinduced increase in ROS production, as well as gene expression of profibrotic and proinflammatory markers [9]. In the present study, we aimed to examine whether the diabetesinduced activation of NOX4-derived ROS specifically in podocytes affects glomerular structure and function in vivo in an animal model of $\mathrm{DN}$, using mice with specific deletion of Nox4 within podocytes.

\section{Methods}

Animals Homozygous floxedNox4 (floxed NADPH oxidase 4) mice (provided by H. H. H. W. Schmidt, CARIM,
Maastricht, the Netherlands) and heterozygous podocytespecific Cre recombinase, podocin-Cre (NPHS2-Cre (podCre)) mice (provided by S. E. Quaggin, Robert H. Lurie Medical Research Center, Chicago, IL, USA) were crossed to generate podocyte-specific, NOX4 knockout (podNox4KO) mice. Cre-mediated recombination resulted in deletion of the Nox4 flanked sequence in the podocyte. We confirmed Nox4 deletion in the podocytes by genotyping (see electronic supplementary material [ESM] Fig. 1a) and qPCR (ESM Fig. 1b), as well as by immunostaining (ESM Fig. 1c). Detailed methods for the isolation of podocytes and glomerular and tubular fractions, as well as confirmation of Nox4 deletion, are provided in ESM Methods. All of the mouse lines were maintained on a $\mathrm{C} 57 \mathrm{BL} / 6$ background. Control mice were littermates of floxedNox4 and podCre mouse crosses.

All animal studies were approved by the Alfred Medical Research \& Education Precinct Animal Ethics Committee under guidelines laid down by the National Health and Medical Research Council of Australia. All animals were housed at the Precinct Animal Centre of the Baker IDI Heart \& Diabetes Institute. During the study, animals had unrestricted access to water and food and were maintained on a $12 \mathrm{~h}$ light/dark cycle in a pathogen-free environment on standard mouse chow (Specialty Feeds, Glen Forest, Perth, WA, Australia).

Induction of diabetes Diabetes was induced in 6-week-old male floxedNox4, podNox $4 \mathrm{KO}$ and podCre mice by five daily i.p. injections of streptozotocin (Sigma-Aldrich, St Louis, $\mathrm{MO}$, USA), at a dose of $55 \mathrm{mg} / \mathrm{kg}$ body weight in citrate buffer, with control mice receiving citrate buffer alone. Only mice with blood glucose $\geq 15 \mathrm{mmol} / \mathrm{l}$ after injection of streptozotocin have been included in experiments; mice with blood glucose $<15 \mathrm{mmol} / 1$ were excluded from the study $(<10 \%$ of the total number of mice). After 10 and 20 weeks, the animals were anaesthetised using sodium pentobarbitone i.p. (100 mg/kg body weight; Euthatal, Sigma-Aldrich, Castle Hill, NSW, Australia). The kidneys were rapidly dissected, weighed and snap-frozen or processed in paraffin for subsequent analysis.

Measurement of metabolic variables At 10 and 20 weeks after induction of diabetes, mice were individually placed into metabolic cages (Iffa Credo, L'Arbresele, France) for $24 \mathrm{~h}$. Urine was collected for subsequent analysis. Blood glucose and glycated haemoglobin were measured, as previously described [9, 20]. Systolic BP was assessed using a computerised non-invasive tail-cuff method [21]. Urinary albumin concentration was measured at 10 and 20 weeks after the induction of diabetes, using a mouse albumin ELISA quantification kit (Bethyl Laboratories, Montgomery, TX, USA). Urinary creatinine was determined using a commercially available creatinine assay kit (Abcam, Cambridge, UK). The urinary albumin/creatinine ratio (ACR) was 
calculated. A mouse cystatin C ELISA kit (BioVendor, Brno, Czech Republic) was used to determine serum cystatin $\mathrm{C}$ according to the manufacturer's instructions.

Histological assessment Kidney sections $(3 \mu \mathrm{m})$ were stained with periodic acid-Schiff for the measurement of glomerulosclerotic injury and mesangial expansion, as well as with Masson's trichrome for the assessment of glomerular ECM accumulation [22]. Mesangial area and ECM accumulation were analysed (percentage of glomerular area) from digital pictures of glomeruli (20 glomeruli per kidney per animal) using Image-Pro Plus 6.0 software (Media Cybernetics, Bethesda, MD, USA), as previously described $[9,20]$. Glomerulosclerotic injury was graded based on the severity of glomerular damage, as previously described [23]. Twenty glomeruli per kidney were assessed in a masked fashion.

In vivo transmission electron microscopy Kidney sections were fixed, embedded, cut and visualised using a Hitachi 7500 transmission electron microscope (Hitachi, Tokyo, Japan). Electron micrographs were used to determine the glomerular basement membrane (GBM) thickness, and the number of filtration slit pores was counted as previously described [24, 25]. For further details, see the ESM Methods.

Immunohistochemistry Immunostaining for collagen IV, fibronectin, nitrotyrosine, nephrin and protein kinase $\mathrm{C}$ (PKC)- $\alpha$ was performed and the proportional area of staining was quantified as previously described [9, 20]. For further details, see the ESM Methods.

Western blot The glomerular fraction was obtained from the frozen renal cortex of the respective control and diabetic mice, as described in ESM Methods. Protein extracts $(5 \mu \mathrm{g})$ from each sample were electrophoresed on $7.5 \%$ acrylamide gels under non-reducing conditions, as previously described [26]. Western blot analysis was then performed with a primary antibody to collagen IV (1:1000, Abcam, Cambridge, MA, USA) and assessed with goat anti-rabbit (Dako, Carpinteria, CA, USA) secondary antibody. Membranes were subsequently probed for $\alpha$-tubulin (Sigma-Aldrich) for determination of equal loading of samples. Blots were detected using the ECL detection kit (Sigma-Aldrich) and densitometry was performed using Quantity One software (Bio-Rad, Richmond, CA, USA).

In vivo glomerular gene expression analysis Total RNA was extracted from isolated glomeruli (Polytron PT-MR2100; Kinematica, Littau/Lucerne, Switzerland) in TRIzol reagent (Invitrogen Australia, Mt Waverely, VIC, Australia), as previously described $[9,20]$. Probes and primer sequences for the RT-PCR of nephrin, vascular endothelial growth factor (VEGF) and monocyte chemoattractant protein (MCP)-1 are described in ESM Table 1. Expression of the genes encoding nephrin, VEGF and MCP-1 was quantified and determined relative to expression of the housekeeping gene 18S (18S ribosomal RNA TaqMan Control Reagent kit) using the TaqMan system (ABI Prism 7500; Perkin-Elmer, Poster City, CA, USA). Results are expressed relative to non-diabetic floxedNox4 mice, which were arbitrarily assigned a value of 1 .

Urinary VEGF ELISA The Quantikine Mouse ELISA kit (R\&D Systems, Minneapolis, MN, USA) was used to measure VEGF in the urine, as per the kit instructions. Urinary VEGF is expressed as picograms per $24 \mathrm{~h}$.

Urinary 8-isoprostanes ELISA An 8-isoprostanes enzyme immunoassay kit (Cayman Chemical Company, Ann Arbor, MI, USA) was used to measure 8-isoprostanes in urine, as described by the manufacturer. Urinary 8 -isoprostanes is expressed as picograms per $24 \mathrm{~h}$.

Urinary 8-hydroxy-2'-deoxyguanosine ELISA A urinary 8-hydroxy-2'-deoxyguanosine (8-OHdG) enzyme immunoassay kit (StressMarq Biosciences Victoria, BC, Canada) was used to measure $8-\mathrm{OHdG}$ in urine, as described by the manufacturer. Urinary 8-OHdG is expressed as nanograms per $24 \mathrm{~h}$.

Table 1 General and metabolic variables after 10 and 20 weeks of study in control and diabetic floxedNox 4 and podNox 4 KO mice ( $n=8-15$ per group)

\begin{tabular}{|c|c|c|c|c|c|c|c|c|}
\hline \multirow[t]{3}{*}{ Variable } & \multicolumn{4}{|l|}{10 weeks } & \multicolumn{4}{|l|}{20 weeks } \\
\hline & \multicolumn{2}{|l|}{ floxedNox4 } & \multicolumn{2}{|c|}{$\operatorname{podNox} 4 \mathrm{KO}$} & \multicolumn{2}{|l|}{ floxedNox4 } & \multicolumn{2}{|c|}{$\operatorname{pod} N o x 4 \mathrm{KO}$} \\
\hline & Control & Diabetes & Control & Diabetes & Control & Diabetes & Control & Diabetes \\
\hline Body weight (g) & $26 \pm 0.8$ & $23 \pm 0.9^{*}$ & $29 \pm 1.1$ & $25 \pm 0.7 *$ & $33 \pm 0.7$ & $26 \pm 0.9^{*}$ & $34 \pm 1.1$ & $27 \pm 1.1^{*}$ \\
\hline Kidney weight/body weight (\%) & $0.54 \pm 0.02$ & $0.70 \pm 0.06^{*}$ & $0.57 \pm 0.01$ & $0.70 \pm 0.03^{*}$ & $0.56 \pm 0.01$ & $0.90 \pm 0.04 *$ & $0.57 \pm 0.01$ & $0.83 \pm 0.03 *$ \\
\hline Systolic BP (mmHg) & $97 \pm 2$ & $99 \pm 1$ & $103 \pm 2$ & $107 \pm 1$ & $98 \pm 3$ & $99 \pm 2$ & $104 \pm 2$ & $105 \pm 2$ \\
\hline Plasma glucose (mmol/l) & $9.3 \pm 0.7$ & $26.5 \pm 3.6^{*}$ & $11.6 \pm 0.7$ & $27.2 \pm 3.4^{*}$ & $9.4 \pm 0.7$ & $28.7 \pm 2.2 *$ & $11.3 \pm 0.6$ & $24.8 \pm 2.3^{*}$ \\
\hline Total glycated haemoglobin (\%) & $6.2 \pm 0.1$ & $10.6 \pm 1.3^{*}$ & $4.5 \pm 0.1$ & $9.1 \pm 0.3^{*}$ & $5.9 \pm 0.8$ & $9.9 \pm 1.1^{*}$ & $6.1 \pm 0.9$ & $10.6 \pm 1.5^{*}$ \\
\hline
\end{tabular}

Data are as means \pm SEM

${ }^{*} p<0.05$ vs the respective control 

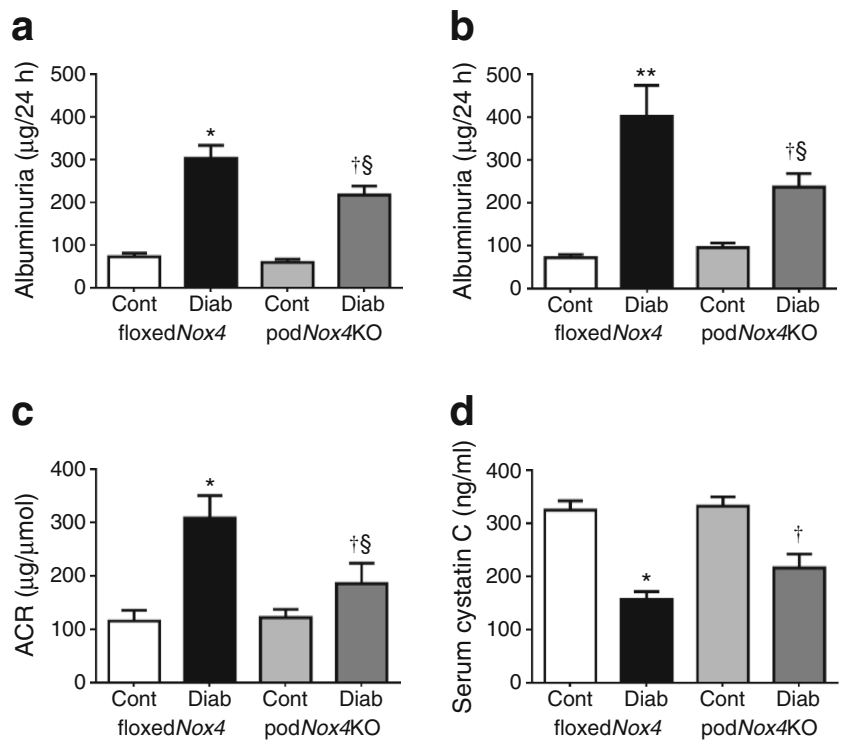

Fig. 1 Podocyte-specific Nox4 deletion attenuated albuminuria in diabetic mice. Urinary albumin excretion at 10 (a) and 20 (b) weeks of diabetes, and ACR (c) and serum cystatin $\mathrm{C}(\mathbf{d})$ at 20 weeks of diabetes in floxedNox 4 and podNox $4 \mathrm{KO}$ mice $(n=10-15$ per group). Data are means \pm SEM. ${ }^{*} p<0.05,{ }^{* *} p<0.01$ vs control (Cont) floxedNox4 mice; ${ }^{\dagger} p<0.05$ vs control (Cont) podNox $4 \mathrm{KO}$ mice; ${ }^{\S} p<0.05$ vs diabetic (Diab) floxedNox4 mice

Lucigenin assays Glomerular fractions obtained from the frozen renal cortices of the different experimental groups were harvested in $100 \mu \mathrm{l}$ ice-cold phosphate buffer $(50 \mathrm{mmol} / \mathrm{l}$
$\mathrm{KH}_{2} \mathrm{PO}_{4}, 1 \mathrm{mmol} / \mathrm{l}$ EGTA and $150 \mathrm{mmol} / 1$ sucrose; $\mathrm{pH}$ 7.4) with protease inhibitors, as previously described [11]. Baseline activity was measured by adding $50 \mu \mathrm{l}$ glomerular extract to $175 \mu \mathrm{l}$ buffer and $2.5 \mu \mathrm{l} 1 \mathrm{mmol} / \mathrm{l}$ lucigenin (Sigma-Aldrich). NADPH-dependent superoxide was measured by the addition of $25 \mu \mathrm{l} 1 \mathrm{mmol} / \mathrm{l} \mathrm{NADPH}$ (Sigma-Aldrich). Baseline activity was subtracted and normalised to the protein concentration.

Statistical analysis All variables were analysed by one-way ANOVA using GraphPad Prism 6 (GraphPad, San Diego, CA, USA) for multiple comparison of the means or by the two-tailed unpaired Mann-Whitney $U$ test, when required. $p<0.05$ was considered to be statistically significant. Results are expressed as mean \pm SEM unless otherwise specified.

\section{Results}

Metabolic variables Induction of diabetes was associated with reduced body weight, elevated blood glucose and increased glycated haemoglobin in both groups when compared with their respective non-diabetic controls (Table 1). Furthermore, no differences in metabolic variables were seen in diabetic podNox4KO mice compared with diabetic floxedNox4 mice (Table 1). In addition, systolic blood
Fig. 2 Podocyte-specific Nox4 deletion attenuated increased VEGF expression and preserved nephrin expression in diabetic mice. Glomerular gene (a) $(n=5-$ 6 per group) and protein $(\mathbf{b}, \mathbf{c})$ ( $n=7-8$ per group) expression of nephrin (magnification $\times 40)$ in respective control (Cont) and diabetic (Diab) floxedNox4 and podNox $4 \mathrm{KO}$ mice after 20 weeks of diabetes. Glomerular Vegf gene expression (d) $(n=5-6$ per group) and urinary VEGF excretion (e) ( $n=9-10$ per group) in respective control and diabetic floxedNox4 and podNox4 $4 \mathrm{KO}$ mice after 20 weeks of diabetes. Data are means \pm SEM. ${ }^{*} p<0.05$ vs control floxedNox4 mice; ${ }^{\star} p=$ 0.05 vs diabetic floxedNox 4 mice; ${ }^{\dagger} p<0.05$ vs control podNox4KO; $\S_{p}<0.05$ vs diabetic floxedNox 4 mice a

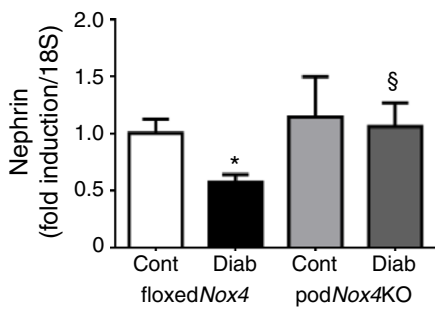

C

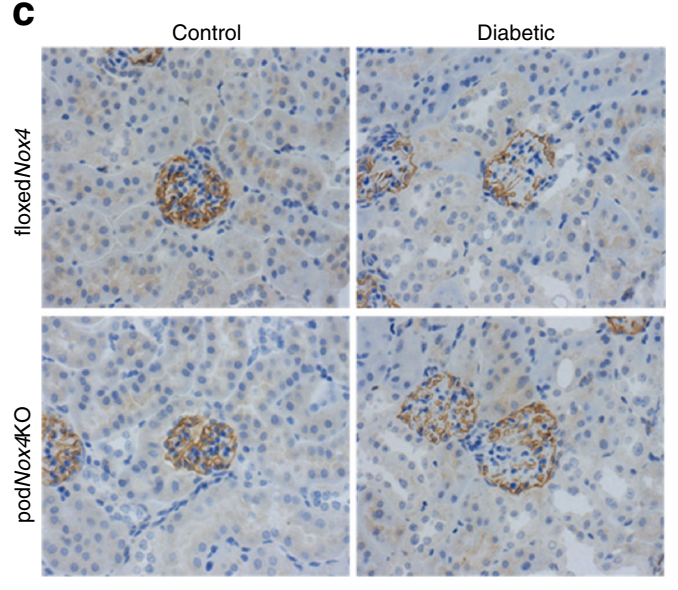

b
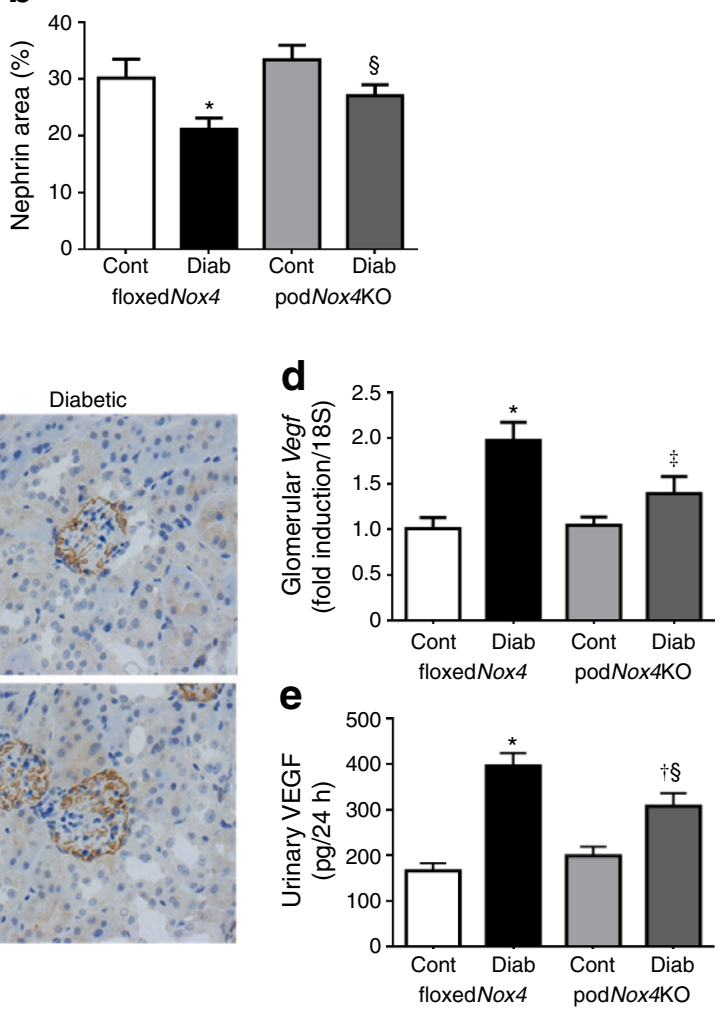
pressure was similar in all groups. The kidney weight/body weight ratio was significantly increased in diabetic mice, with similar ratios in diabetic floxedNox4 and podNox $4 \mathrm{KO}$ mice. Furthermore, metabolic variables were unchanged in podCre mice when compared with floxedNox4 mice after 20 weeks of diabetes (ESM Table 2).

Renal functional variables Albuminuria was significantly attenuated after 10 and 20 weeks of diabetes in podNox4KO mice when compared with diabetic floxedNox4 mice (Fig. 1a, b). Similar effects were observed when the data were expressed as urinary ACR after 20 weeks of diabetes (Fig. 1c). Furthermore, no difference in albuminuria was found in podCre mice when compared with floxedNox4 mice after 20 weeks of diabetes (ESM Table 2). The serum cystatin $\mathrm{C}$ level was reduced in both diabetic floxedNox4 and podNox4KO mice when compared with their non-diabetic counterparts (Fig. 1d), suggesting that the glomerular filtration rate was not reduced by Nox4 deletion in podocytes and that both groups of diabetic mice exhibited hyperfiltration.

Gene and protein expression of nephrin As previously demonstrated in another model of DN [27], we found decreased gene and protein expression of glomerular nephrin in diabetic floxedNox4 mice (Fig. 2a-c). Interestingly, the diabetes-induced decrease in nephrin expression was preserved in diabetic podNox4KO mice (Fig. 2a-c).

Gene expression and urinary excretion of VEGF We observed that glomerular Vegf (also known as Vegfa) gene expression and urinary excretion of VEGF were higher in diabetic floxedNox4 mice when compared with nondiabetic controls, and these variables were attenuated in diabetic podNox4KO mice (Fig. 2d, e).

Glomerular structural assessment Glomerulosclerosis and mesangial area (Fig. 3a-c) as well as ECM accumulation (Fig. 3d, e) were increased in floxedNox 4 mice after 20 weeks of diabetes when compared with non-diabetic floxedNox4 mice. Increased glomerulosclerosis and mesangial area $(p<0.05)$ as well as ECM accumulation $(p=0.05)$ were attenuated in diabetic podNox4KO mice (Fig. 3a-e).

GBM thickness and podocyte foot process effacement Using quantifying histomorphometric techniques, both thickening of GBM as well as irregularity in podocyte foot process
Fig. 3 Podocyte-specific Nox4 deletion attenuated glomerular injury in diabetic mice. Periodic acid-Schiff staining (magnification $\times 40)(\mathbf{a})$, glomerulosclerotic index (b) and mesangial area expansion (c), as well as Masson's trichrome staining $($ magnification $\times 40)(\mathbf{d})$ and glomerular ECM accumulation (e) in control (Cont) and diabetic (Diab) floxedNox4 and podNox4KO mice $(n=7-10$ per group) after 20 weeks of diabetes. Data are means $\pm \mathrm{SEM}$. ${ }^{*} p<0.05$ vs control floxedNox4 mice; ${ }^{\ddagger} p=0.05$ vs diabetic floxedNox 4 mice; ${ }^{\dagger} p<0.05$ vs control podNox $4 \mathrm{KO} ;{ }^{\S} p<0.05$ vs diabetic floxedNox4 mice a

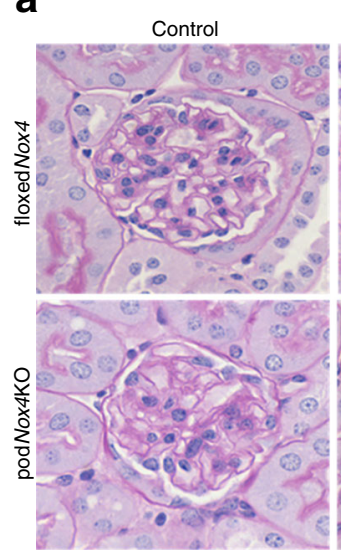

d

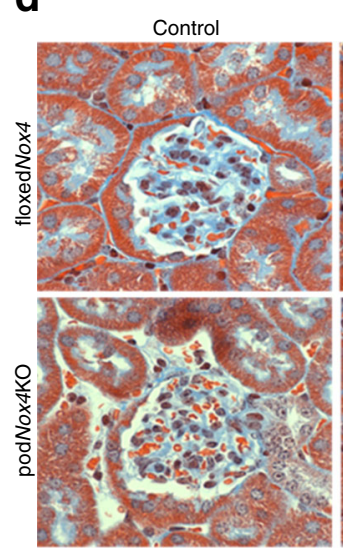

b
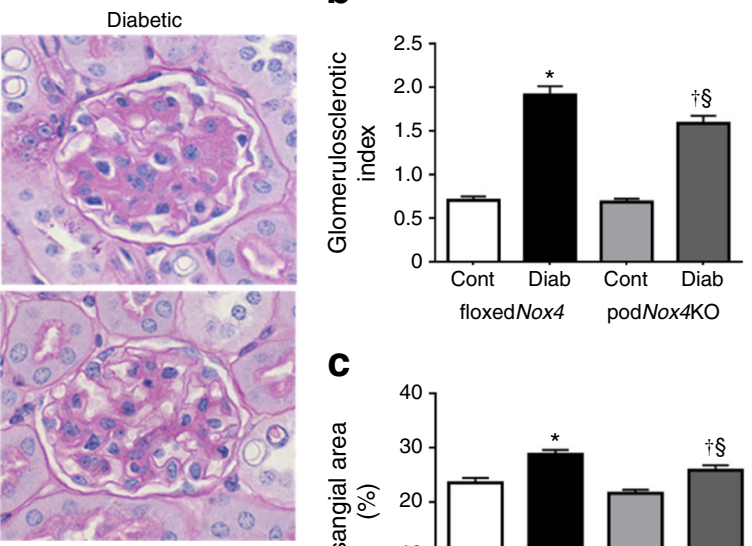

C

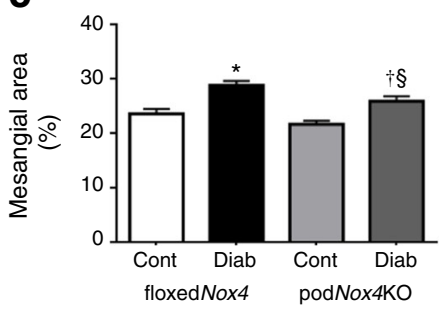

e

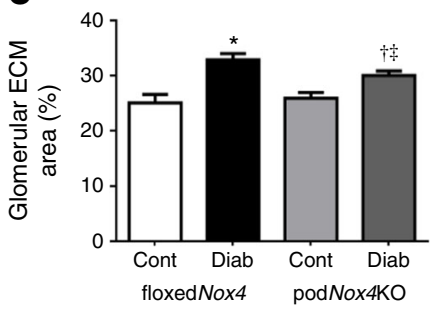


dimensions and unevenly spread filtration slit pores suggestive of foot process effacement were seen in diabetic floxedNox4 mice when compared with control floxedNox4 mice (Fig. 4a, b). Furthermore, diminution in the number of filtration slit pores per $100 \mu \mathrm{m}$ of GBM $(p<0.05)$ was seen in diabetic floxedNox4 mice, indicative of foot process broadening (Fig. 4c). Interestingly, podocyte-specific NOX4-deficient diabetic mice appeared to have decreased GBM thickness, less podocyte foot process effacement and a high number of filtration slit pores when compared with diabetic floxedNox4 mice (Fig. 4a-c).

Glomerular ECM proteins We examined glomerular collagen IV and fibronectin accumulation in diabetic mice. Consistent with the findings on glomerulosclerosis and mesangial expansion, collagen IV protein production was significantly increased in the glomeruli after 20 weeks of diabetes in floxedNox 4 mice when compared with non-diabetic floxedNox4 controls, as assessed by immunohistochemistry (Fig. 5a, b) or western blot (Fig. 5c, d). Importantly, the

a

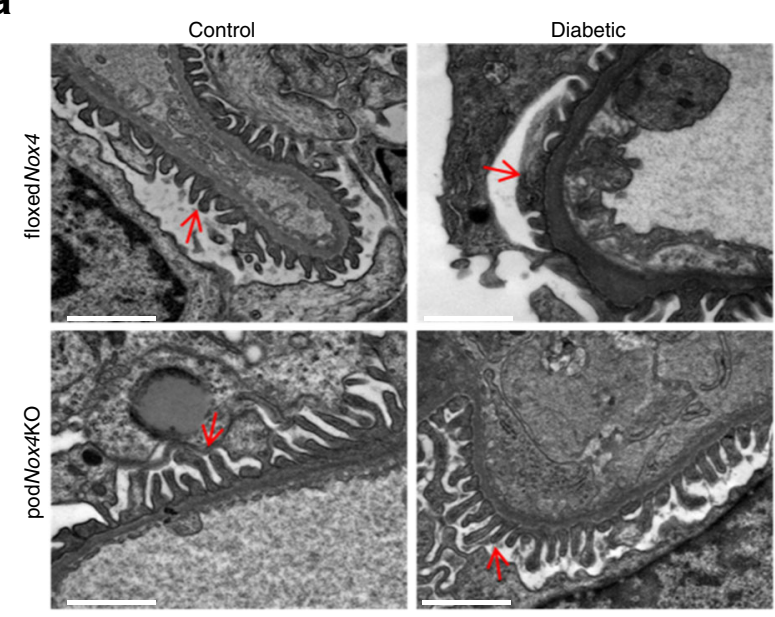

b
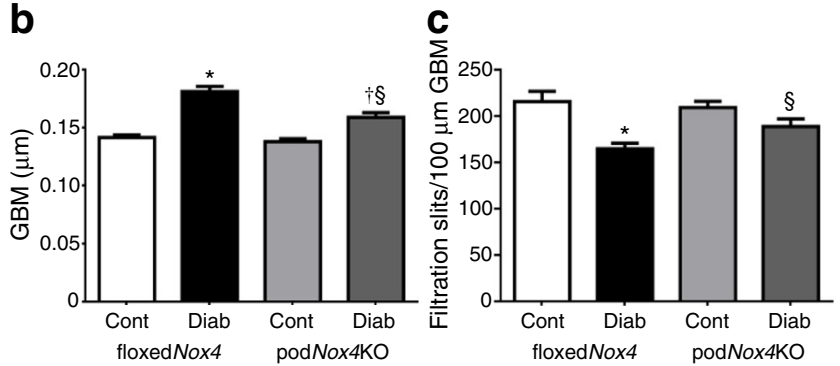

Fig. 4 Podocyte-specific Nox4 deletion attenuated GBM thickness and filtration slit pores in diabetic mice. Transmission electron micrographs for GBM thickness $(\mathbf{a}, \mathbf{b})$, podocyte foot process effacement (a) and the number of slits per $100 \mu \mathrm{m}$ of GBM (c) in respective control (Cont) and diabetic (Diab) floxedNox4 and podNox4KO mice after 20 weeks of diabetes. Red arrows indicate podocyte foot processes. Images were taken at $\times 30,000$ magnification. Scale bar, $0.5 \mu \mathrm{m}$. Data are means $\pm \mathrm{SEM}$. ${ }^{*} p<0.05$ vs control floxedNox 4 mice; ${ }^{\dagger} p<0.05$ vs control podNox $4 \mathrm{KO}$; $\S_{p}<0.05$ vs diabetic floxedNox 4 mice increase in collagen IV production was attenuated in podNox4KO mice (Fig. 5a-d). Similarly, fibronectin protein accumulation was increased in the glomeruli of floxedNox4 mice after 20 weeks of diabetes and this variable was attenuated in podocyte-specific NOX4-deficient mice (Fig. 5e, f).

Oxidative stress markers Urinary excretion of 8-isoprostane and 8-OHdG were increased in diabetic floxedNox4 mice compared with non-diabetic controls (Fig. 6a, b). The diabetesinduced increases in urinary level of 8-isoprostane and 8-OHdG were attenuated in podocyte-specific NOX4-deficient diabetic mice (Fig. 6a, b). To strengthen the findings on renal ROS levels, we next examined NADPH-dependent glomerular superoxide generation, as well as immunostaining of glomerular nitrotyrosine. Glomerular superoxide and nitrotyrosine accumulation were increased in floxedNox4 mice after 20 weeks of diabetes and attenuated in diabetic podNox $4 \mathrm{KO}$ mice (Fig. 6c-e). To study the effect of NOX4 on $\mathrm{H}_{2} \mathrm{O}_{2}$ production, we examined podocytes with and without Nox4 expression, and found that silencing of Nox4 using Nox4 shRNAin human podocytes resulted in attenuation of increased levels of $\mathrm{H}_{2} \mathrm{O}_{2}$ generation in response to high glucose (ESM Fig. 2).

Glomerular PKC- $\alpha$ and MCP-1 We examined glomerular PKC- $\alpha$ expression and found increased production of this PKC isoform in diabetic floxedNox4 mice when compared with non-diabetic controls (Fig. 7a, b). Furthermore, glomerular PKC- $\alpha$ production was attenuated in diabetic

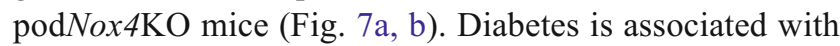
increased inflammation [28]. Indeed, we observed increased glomerular $\mathrm{Mcp}-1$ (also known as $\mathrm{Ccl}$ ) expression in diabetic floxedNox4 mice when compared with non-diabetic controls (Fig. 7c). This inflammatory variable was attenuated in diabetic podNox $4 \mathrm{KO}$ mice $(p=0.05)$ (Fig. 7c).

\section{Discussion}

This study provides, for the first time, clear evidence that podocyte-specific Nox4 deletion affords renoprotection in vivo in a mouse model of DN. Previously it has been shown by our group that global genetic deletion of Nox4 prevented the development of albuminuria and glomerular injury via a reduction in renal ROS in diabetic mice [9]. To further address this issue and to specifically determine the potential mechanisms of renoprotection at a cellular level, an in vitro study involving silencing of Nox4 in human podocytes was performed. Indeed, knockdown of NOX4 in human podocytes reduced high-glucose-induced ROS production, as well as various markers of fibrosis and inflammation. These findings, albeit in vitro, suggested a deleterious effect of NOX4 within podocytes, potentially attenuating key pathways implicated in 
Fig. 5 Podocyte-specific Nox4 deletion attenuated glomerular collagen IV and fibronectin expression in diabetic mice. Immunostaining of collagen IV (a, b) and fibronectin $(\mathbf{e}, \mathbf{f})(n=7-8$ per group) (magnification $\times 40$ ) as well as western blotting for collagen IV (c, d) ( $n=6$ per group) in the glomeruli of respective control (Cont) and diabetic (Diab) floxedNox4 and podNox4KO mice after 20 weeks of diabetes. Data are means \pm SEM. $* p<0.05$ vs control floxedNox4 mice; ${ }^{\S} p<0.05$ vs diabetic floxedNox4 mice

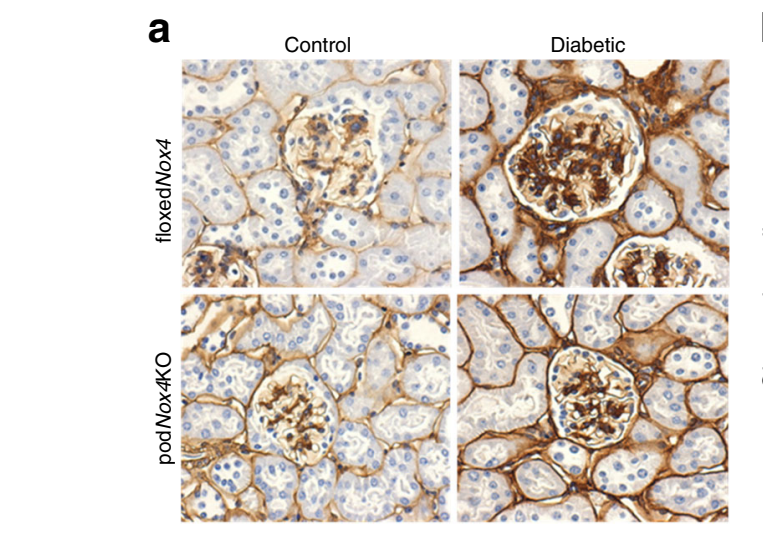

b
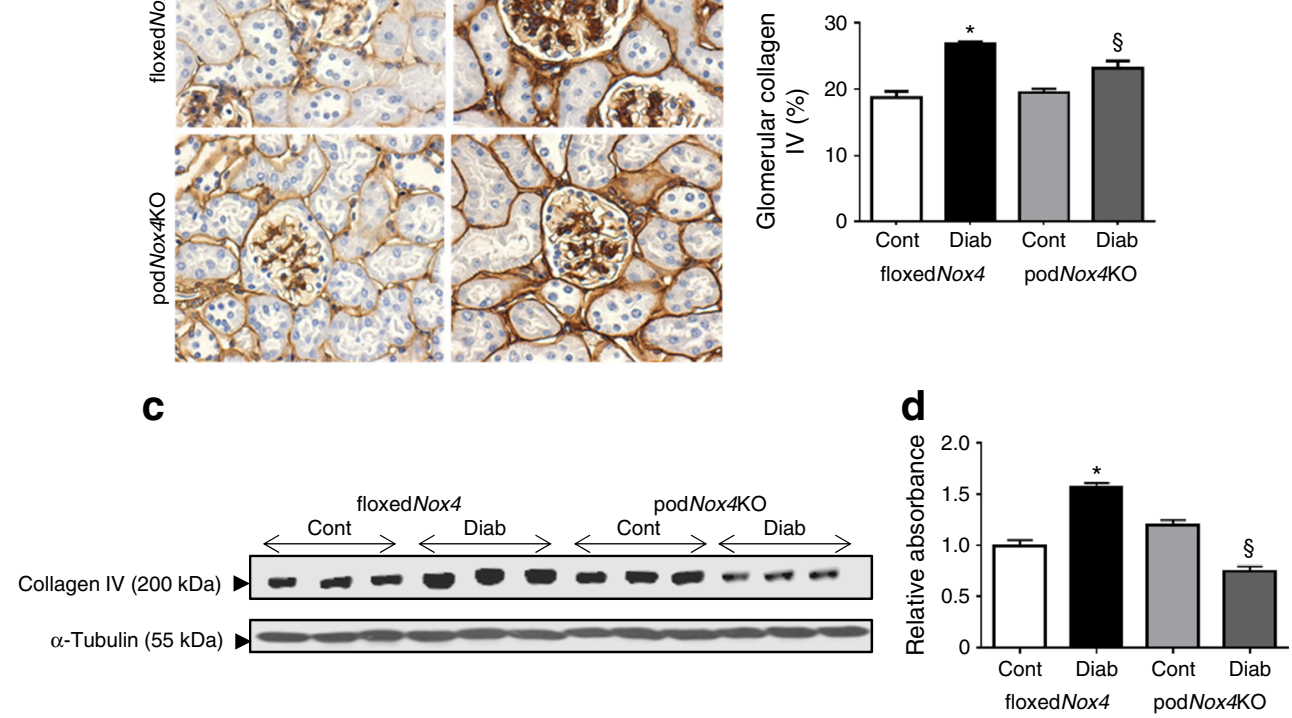

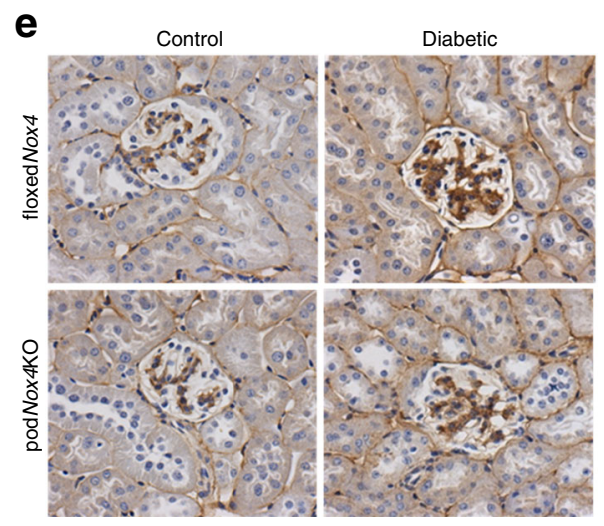

f

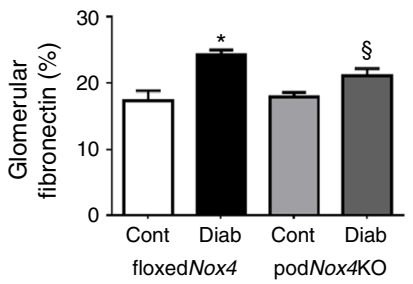

the development of albuminuria and renal injury in diabetes [9]. To translate these findings in podocytes to an in vivo context, the present study addressed and indeed demonstrated that podocyte-specific Nox4 deletion attenuates the diabetes-induced increase in albuminuria by approximately $50 \%$ in diabetic podNox $4 \mathrm{KO}$ mice. This finding strongly supports the view that NOX4-derived ROS in the podocyte plays a crucial role in the regulation of albuminuria in diabetes. With identification of the prevention of the diabetes-induced decrease in nephrin expression and reduced podocyte foot process effacement in the podNox4KO diabetic mice, this would be consistent with a deleterious effect of NOX4 in podocytes, consistent with the previous in vitro studies [9]. Indeed, changes in the expression of the podocyte-specific marker nephrin and podocyte damage, including podocyte foot process effacement, have been shown to correlate with albuminuria in diabetes [24, 27, 29].
To further explore potential mechanisms linking NOX4 to renal injury, renal and urinary expression of VEGF were examined, this growth factor having previously been reported to be linked to the development of albuminuria, particularly in the setting of diabetes [30-33]. We have previously reported a decrease in VEGF expression in the glomeruli of diabetic global NOX4 knockout mice, as well as in Nox4 silenced human podocytes in response to high glucose [9]. Indeed, in this study, we observed a reduction in urinary VEGF excretion as well decreased glomerular VEGF expression in diabetic

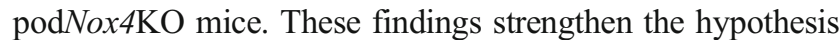
that NOX4-derived ROS play a crucial role in modulating and regulating VEGF expression in podocytes. Interestingly, podocyte-specific Nox4 deletion also attenuated various glomerular ultra-structural changes, including glomerulosclerosis, mesangial expansion and accumulation of ECM proteins, including collagen IV and fibronectin, in diabetic podNox $4 \mathrm{KO}$ mice. Abrogation of glomerular 
Fig. 6 Podocyte-specific Nox4 deletion attenuated renal ROS in diabetic mice. Urinary 8isoprostane (a) and 8-OHdG excretion (b) $(n=8-10$ per group) and immunostaining for glomerular nitrotyrosine $(\mathbf{c}, \mathbf{d})$ (magnification $\times 40)(n=6-8$ per group) and NADPH-dependent lucigenin assay (e) $(n=6)$ in respective control (Cont) and diabetic (Diab) floxedNox4 and podNox4KO mice after 20 weeks of diabetes. Data are means \pm SEM. ${ }^{*} p<0.05$ vs control floxedNox4 mice; ${ }^{\dagger} p<0.05$ vs control podNox $4 \mathrm{KO} ;{ }^{\star} p=0.05$ vs diabetic floxedNox4 mice; ${ }^{\S} p<0.05$ vs diabetic floxedNox4 mice
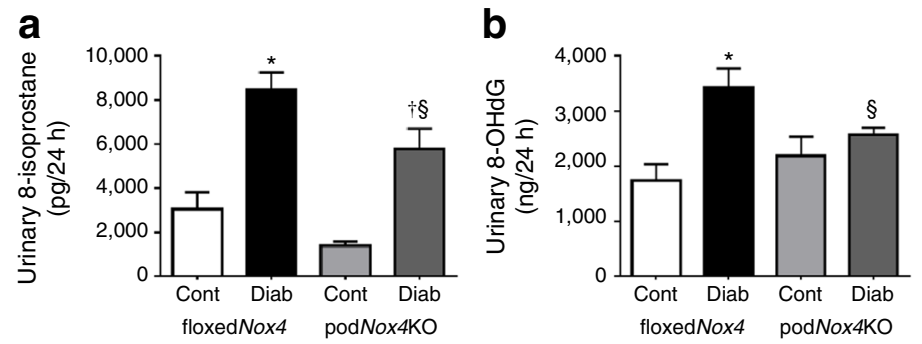

C
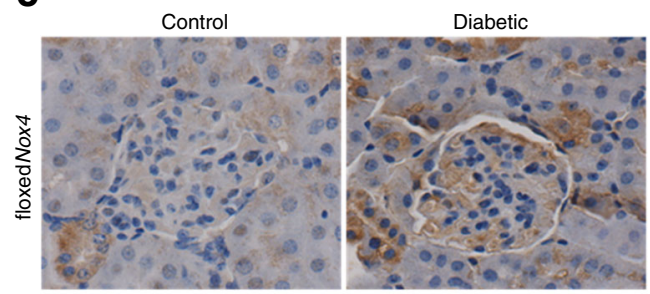

d

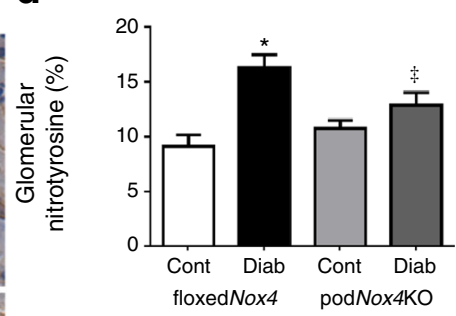

e

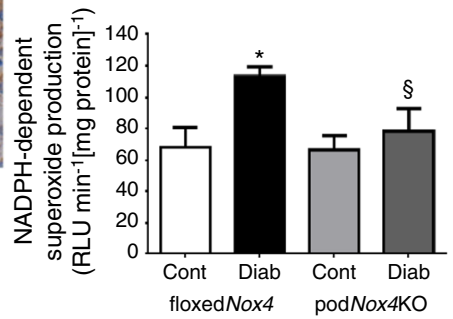

structural damage in diabetic podNox4 $\mathrm{KO}$ mice would indicate potential cross-talk between podocytes and other glomerular cells that are involved in the process of glomerulosclerosis. A recent study, albeit in the heart, supports our findings that NOX4 plays a role in fibrosis and hypertrophy [34]. In that study, NOX4 was shown to induce cardiac fibrosis and hypertrophy via activation of the Akt/mTOR and nuclear factor- $\mathrm{kB}$ signalling pathways [34].
A correlation between albuminuria and GBM thickness has been reported previously $[35,36]$. Thickening of the GBM is considered to be one of the characteristic lesions in diabetic patients with albuminuria [35, 37]. It has been shown that GBM thickening in diabetes occurs as a consequence of accumulation of increased ECM components [38, 39]. The accumulation of ECM proteins results from both increased production and decreased degradation of these proteins. Indeed,
Fig. 7 Podocyte-specific Nox4 deletion attenuated glomerular PKC- $\alpha$ and $M c p-1$ expression in diabetic mice. Immunostaining for PKC- $\alpha$ in glomeruli $(n=8-10$ per group) (a, b) (magnification $\times 40)$ and gene expression of glomerular Mcp-1 (c) in respective control (Cont) and diabetic (Diab) floxedNox4 and $\operatorname{podNox} 4 \mathrm{KO}$ mice after 20 weeks of diabetes. Data are means \pm SEM. ${ }^{*} p<0.05$ vs control floxedNox4 mice; ${ }^{\ddagger} p=0.05$ vs diabetic floxedNox 4 mice; ${ }_{p}<0.01$ vs diabetic floxedNox4 mice a

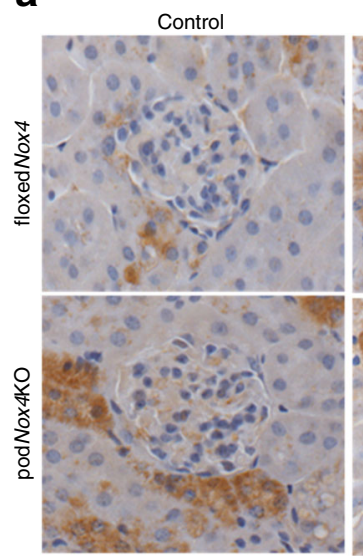

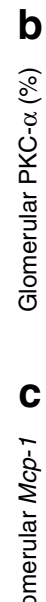

C
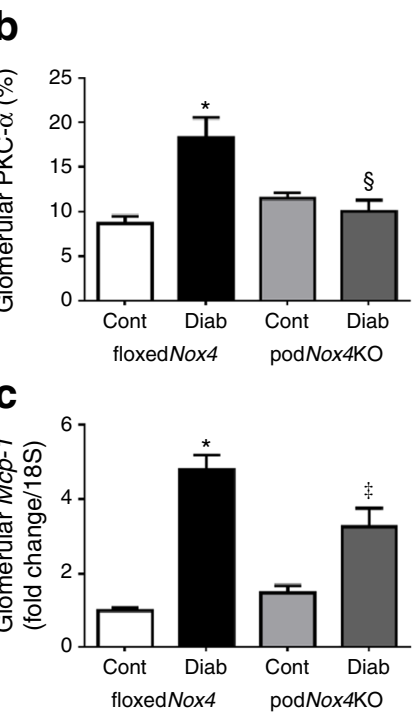
increased GBM thickness and accumulation of ECM proteins (collagen IV and fibronectin) were seen in diabetic mice and these changes were attenuated in diabetic podNox4KO mice, consistent with NOX4 in podocytes playing a central role in regulating the production of ECM proteins. Another key morphological feature of DN, identified using electron microscopy, is a reduction in the number of slit pores per unit length of GBM, which reflects foot process broadening, as has been shown in diabetic rodents [24]. This diabetes-associated reduction in filtration slit pores was attenuated in diabetic podNox4 $4 \mathrm{KO}$ mice, further emphasising the importance of NOX4 in promoting podocytopathy, as seen in the diabetic kidney.

Since it is considered that NOX4, also known as 'renox', is a key source of renal ROS, this study used various approaches to evaluate renal ROS generation. Markers of DNA oxidation (8-OHdG) and lipid peroxidation (8-isoprostane) have been shown to be increased in the urine in experimental models of diabetes [32, 40, 41]. In addition, increased urinary levels of 8-OHdG have been reported to be associated with loss of podocytes in diabetic rodents [40]. Thus, attenuation of urinary excretion of the oxidative stress markers 8-isoprostane and $8-\mathrm{OHdG}$, as well as glomerular superoxide and nitrotyrosine, in diabetic podNox4KO mice, as seen in this study, supports the view that NOX4-derived ROS mediate podocyte damage and ultimately other markers of renal injury. The ability of Nox4 deletion only in podocytes to significantly reduce a range of markers of glomerular and renal ROS emphasises the importance of podocyte NOX4 per se as a source of ROS in the diabetic kidney.

There is a close interrelationship between ROS and certain $\mathrm{PKC}$ isoforms in the development and progression of DN [32, 42]. Recently, it has been shown that NOX4-derived ROS can activate certain PKC isoforms, including PKC- $\alpha$, within the kidney, thereby promoting renal injury in experimental diabetes [32]. Thus, we examined the association of podocytespecific NOX4 with PKC- $\alpha$, which has been the most closely identified isoform with the development of albuminuria, nephrin depletion and upregulation of intrarenal VEGF in diabetes $[43,44]$. Indeed, we found that the diabetes-induced increase in glomerular PKC- $\alpha$ expression was attenuated in diabetic podNox4KO mice. Quack et al have demonstrated that acute hyperglycaemia increases nephrin endocytosis in a PKC- $\alpha$-dependent manner [45] and this effect is considered to promote albuminuria. In this study, we found similar changes in VEGF and nephrin expression as well as in albuminuria, in diabetic podNox4KO mice to those seen in diabetic $P k c \alpha \mathrm{KO}$ mice, and also noted a reduction in glomerular PKC- $\alpha$ expression in podNox4KO mice. Thus, we postulate that NOX4 is a key upstream regulator of PKC- $\alpha$ expression in the setting of diabetes. Indeed, the renoprotective effects of Nox4 deletion could represent a new approach to target the deleterious effects of PKC- $\alpha$ in the diabetic kidney. In addition, the diabetes-induced increase in glomerular $M c p-1$ expression was found to be attenuated in podocyte-specific NOX4-deficient diabetic mice. This would indicate that targeting NOX4 in the podocytes not only reduces podocytopathy and glomerular ECM accumulation, but might also play a key role in attenuating intrarenal inflammation.

The findings of this study build on an increasing amount of data implicating NOX4 as a potential target for renoprotection. The advent of new agents that target NOX4, such as GKT137831, provide an opportunity to test whether pharmacological inhibition of NOX4 can be renoprotective. Although GKT137831 is not specific for the NOX4 isoform, several groups, including our own, have demonstrated renal benefits with this agent in various experimental models of DN $[9,46]$.

In conclusion, NOX4 within the podocyte appears to be a major contributor to renal ROS generation, activation of PKC- $\alpha$, enhanced intrarenal fibrosis and inflammation, as well as increasing VEGF and decreasing nephrin expression. Furthermore, NOX4 plays a pivotal role in influencing podocyte ultrafiltration, with a subsequent impact on glomerular structure and albuminuria, hallmarks of DN. Hence, targeting NOX4 specifically in the podocyte might provide a new therapeutic approach in preventing the progression of diabetic kidney disease.

Acknowledgements The authors thank M. Arnstein, K. Gilbert, S. Sacca, E. Lastavec, M. Haillay (Baker IDI Heart \& Diabetes Institute, Melbourne, VIC, Australia) for experimental animal handling and technical support.

\section{Compliance with ethical standards}

Funding This work was supported by the National Health \& Medical Research Council of Australia (NHMRC), a JDRF Program/Project Grant, the Diabetes Australia Research Trust and the FP7 framework programme. KAJ-D is supported by a NHMRC Senior Research Fellowship and MEC is a Senior Principal Research Fellow for the NHMRC. HHHWS is supported by a Marie Curie International Reintegration Grant, an ERC Advanced Investigator Grant and the Dutch Kidney Foundation.

Duality of interest statement The authors declare that there is no duality of interest associated with this manuscript.

Contribution statement All authors contributed to the study concept and design, and the interpretation of the data. JCJ collected research data, contributed to discussions and wrote, reviewed and edited the manuscript. VT-B, CB, SPG and BSMC collected and analysed the data and contributed to drafting the manuscript. GR helped with electron microscopy data and contributed to drafting the manuscript. SEQ and MEC contributed in discussions, and reviewed and edited the manuscript. HHHWS and KAJ-D provided intellectual content in writing the discussions, and reviewed and edited the manuscript. All authors revised the article and approved the final version for publication. JCJ and KAJ-D are the guarantors of this work and, as such, had full access to all of the data in the study and take responsibility for the integrity of the data and the accuracy of the data analysis. 


\section{References}

1. Roglic G, Unwin N, Bennett PH et al (2005) The burden of mortality attributable to diabetes: realistic estimates for the year 2000 . Diabetes Care 28:2130-2135

2. Molitch ME, DeFronzo RA, Franz MJ et al (2004) Nephropathy in diabetes. Diabetes Care 27(Suppl 1):S79-s83

3. Bjorn SF, Bangstad HJ, Hanssen KF et al (1995) Glomerular epithelial foot processes and filtration slits in IDDM patients. Diabetologia 38:1197-1204

4. Eid AA, Gorin Y, Fagg BM et al (2009) Mechanisms of podocyte injury in diabetes: role of cytochrome P450 and NADPH oxidases. Diabetes 58:1201-1211

5. Tryggvason K, Patrakka J, Wartiovaara J (2006) Hereditary proteinuria syndromes and mechanisms of proteinuria. N Engl J Med 354: 1387-1401

6. Calcutt NA, Cooper ME, Kern TS, Schmidt AM (2009) Therapies for hyperglycaemia-induced diabetic complications: from animal models to clinical trials. Nat Rev Drug Discov 8:417-429

7. Gill PS, Wilcox CS (2006) NADPH oxidases in the kidney. Antioxid Redox Signal 8:1597-1607

8. Kaneto H, Katakami N, Kawamori D et al (2007) Involvement of oxidative stress in the pathogenesis of diabetes. Antioxid Redox Signal 9:355-366

9. Jha JC, Gray SP, Barit D et al (2014) Genetic targeting or pharmacologic inhibition of NADPH oxidase nox 4 provides renoprotection in long-term diabetic nephropathy. J Am Soc Nephrol 25:1237-1254

10. Gorin Y, Block K, Hernandez J et al (2005) Nox4 NAD(P)H oxidase mediates hypertrophy and fibronectin expression in the diabetic kidney. J Biol Chem 280:39616-39626

11. Holterman CE, Thibodeau JF, Towaij C et al (2014) Nephropathy and elevated BP in mice with podocyte-specific NADPH oxidase 5 expression. J Am Soc Nephrol 25:784-797

12. Brenner BM, Hostetter TH, Humes HD (1978) Molecular basis of proteinuria of glomerular origin. N Engl J Med 298:826-833

13. Palicz A, Foubert TR, Jesaitis AJ, Marodi L, McPhail LC (2001) Phosphatidic acid and diacylglycerol directly activate NADPH oxidase by interacting with enzyme components. J Biol Chem 276: 3090-3097

14. Satoh M, Fujimoto S, Haruna Y et al (2005) NAD(P)H oxidase and uncoupled nitric oxide synthase are major sources of glomerular superoxide in rats with experimental diabetic nephropathy. Am J Physiol Ren Physiol 288:F1144-F1152

15. Ding G, Reddy K, Kapasi AA et al (2002) Angiotensin II induces apoptosis in rat glomerular epithelial cells. Am J Physiol Ren Physiol 283:F173-F180

16. Schiffer M, Bitzer M, Roberts IS et al (2001) Apoptosis in podocytes induced by TGF- $\beta$ and Smad7. J Clin Invest 108:807816

17. Wolf G, Chen S, Ziyadeh FN (2005) From the periphery of the glomerular capillary wall toward the center of disease: podocyte injury comes of age in diabetic nephropathy. Diabetes 54:16261634

18. Asaba K, Tojo A, Onozato ML et al (2005) Effects of NADPH oxidase inhibitor in diabetic nephropathy. Kidney Int 67:18901898

19. Susztak K, Raff AC, Schiffer M, Bottinger EP (2006) Glucoseinduced reactive oxygen species cause apoptosis of podocytes and podocyte depletion at the onset of diabetic nephropathy. Diabetes $55: 225-233$

20. Watson AM, Li J, Schumacher C et al (2010) The endothelin receptor antagonist avosentan ameliorates nephropathy and atherosclerosis in diabetic apolipoprotein E knockout mice. Diabetologia 53:192-203
21. Krege JH, Hodgin JB, Hagaman JR, Smithies O (1995) A noninvasive computerized tail-cuff system for measuring blood pressure in mice. Hypertension 25:1111-1115

22. Chai Z, Dai A, Tu Y et al (2013) Genetic deletion of cell division autoantigen 1 retards diabetes-associated renal injury. J Am Soc Nephrol 24:1782-1792

23. Lassila M, Seah KK, Allen TJ et al (2004) Accelerated nephropathy in diabetic apolipoprotein e-knockout mouse: role of advanced glycation end products. J Am Soc Nephrol 15:2125-2138

24. Mifsud SA, Allen TJ, Bertram JF et al (2001) Podocyte foot process broadening in experimental diabetic nephropathy: amelioration with renin-angiotensin blockade. Diabetologia 44:878-882

25. Sato S, Sasaki Y, Adachi A, Ghazizadeh M (2010) Validation of glomerular basement membrane thickness changes with aging in minimal change disease. Pathobiology 77:315-319

26. Koulis C, Chow BS, McKelvey M et al (2015) AT2R agonist, compound 21, is reno-protective against type 1 diabetic nephropathy. Hypertension 65:1073-1081

27. Doublier S, Salvidio G, Lupia E et al (2003) Nephrin expression is reduced in human diabetic nephropathy: evidence for a distinct role for glycated albumin and angiotensin II. Diabetes 52:1023-1030

28. Chow FY, Nikolic-Paterson DJ, Ozols E, Atkins RC, Rollin BJ, Tesch GH (2006) Monocyte chemoattractant protein-1 promotes the development of diabetic renal injury in streptozotocin-treated mice. Kidney Int 69:73-80

29. Cooper ME, Mundel P, Boner G (2002) Role of nephrin in renal disease including diabetic nephropathy. Semin Nephrol 22:393398

30. Cooper ME, Vranes D, Youssef S et al (1999) Increased renal expression of vascular endothelial growth factor (VEGF) and its receptor VEGFR-2 in experimental diabetes. Diabetes 48:2229-2239

31. Sung SH, Ziyadeh FN, Wang A, Pyagay PE, Kanwar YS, Chen S (2006) Blockade of vascular endothelial growth factor signaling ameliorates diabetic albuminuria in mice. J Am Soc Nephrol 17: 3093-3104

32. Thallas-Bonke V, Jha JC, Gray SP et al (2014) Nox-4 deletion reduces oxidative stress and injury by PKC- $\alpha$-associated mechanisms in diabetic nephropathy. Physiol Rep 2, e12192

33. Ziyadeh FN, Wolf G (2008) Pathogenesis of the podocytopathy and proteinuria in diabetic glomerulopathy. Curr Diabetes Rev 4:39-45

34. Zhao QD, Viswanadhapalli S, Williams P et al (2015) NADPH oxidase 4 induces cardiac fibrosis and hypertrophy through activating Akt/mTOR and NFKB signaling pathways. Circulation 131: 643-655

35. Bangstad HJ, Osterby R, Dahl-Jorgensen K, Berg KJ, Hartmann A, Hanssen KF (1994) Improvement of blood glucose control in IDDM patients retards the progression of morphological changes in early diabetic nephropathy. Diabetologia 37:483-490

36. Tervaert TW, Mooyaart AL, Amann K et al (2010) Pathologic classification of diabetic nephropathy. J Am Soc Nephrol 21:556-563

37. Mac-Moune Lai F, Szeto CC, Choi PC et al (2004) Isolate diffuse thickening of glomerular capillary basement membrane: a renal lesion in prediabetes? Mod Pathol 17:1506-1512

38. Falk RJ, Scheinman JI, Mauer SM, Michael AF (1983) Polyantigenic expansion of basement membrane constituents in diabetic nephropathy. Diabetes 32(Suppl 2):34-39

39. Kim Y, Kleppel MM, Butkowski R, Mauer SM, Wieslander J, Michael AF (1991) Differential expression of basement membrane collagen chains in diabetic nephropathy. Am J Pathol 138:413-420

40. Kim J, Shon E, Kim CS, Kim JS (2012) Renal podocyte injury in a rat model of type 2 diabetes is prevented by metformin. Exp Diabetes Res 2012:210821

41. Montero A, Munger KA, Khan RZ et al (2000) $F_{2}$-isoprostanes mediate high glucose-induced TGF- $\beta$ synthesis and glomerular proteinuria in experimental type I diabetes. Kidney Int 58:19631972 
42. Giorgi C, Agnoletto C, Baldini C et al (2010) Redox control of protein kinase C: cell- and disease-specific aspects. Antioxid Redox Signal 13:1051-1085

43. Menne J, Meier M, Park JK et al (2006) Nephrin loss in experimental diabetic nephropathy is prevented by deletion of protein kinase $\mathrm{C}$ alpha signaling in-vivo. Kidney Int 70:1456-1462

44. Menne J, Shushakova N, Bartels J et al (2013) Dual inhibition of classical protein kinase $\mathrm{C}-\alpha$ and protein kinase $\mathrm{C}-\beta$ isoforms protects against experimental murine diabetic nephropathy. Diabetes 62:1167-1174

45. Quack I, Woznowski M, Potthoff SA et al (2011) PKC $\alpha$ mediates $\beta$-arrestin2-dependent nephrin endocytosis in hyperglycemia. $\mathrm{J}$ Biol Chem 286:12959-12970

46. Gorin Y, Cavaglieri RC, Khazim K et al (2015) Targeting NADPH oxidase with a novel dual Nox1/Nox4 inhibitor attenuates renal pathology in type 1 diabetes. Am J Physiol Ren Physiol 308: F1276-F1287 